\title{
Protection of User-Defined Sensitive Attributes on Online Social Networks against Attribute Inference Attack via Adversarial Data Mining
}

\author{
Khondker Jahid Reza ${ }^{1}$, Md Zahidul Islam ${ }^{1}$, and Vladimir Estivill-Castro ${ }^{2}$ \\ 1 School of Computing and Mathematics, Charles Sturt University, \\ Panorama Avenue, Bathurst 2795, NSW, Australia. \\ 2 Departament de Tecnologies de la Informació i les Comunicacions, \\ Universitat Pompeu Fabra, Roc Boronat, 138, 08018 Barcelona, Spain \\ \{kreza,zislam\}@csu.edu.au, vladimir.estivill@upf.edu
}

\begin{abstract}
Online social network (OSN) users share various types of personal information with other users. By analysing such personal information, a malicious data miner (or an attacker) can infer the sensitive information about the user which has not been disclosed publicly. This is generally known as attribute inference attack. In this study, we propose a privacy preserving technique, namely $3 L P+$, that can protect users' multiple sensitive information from being inferred. We experimentally show that the $3 L P+$ algorithm can provide better privacy than an existing technique while maintaining the utility of users' data.
\end{abstract}

Keywords: Attribute inference - Data mining · Online social networks - Privacy protection technique.

\section{Introduction}

Online social networks (OSNs) data can be used to automatically and accurately predict a range of highly sensitive personal attributes including: sexual orientation, ethnicity, religious and political views, personality traits, intelligence, happiness, use of addictive substances, and parental separation [13]. Participants of OSNsmay wish to keep some information-items confidential, but the attributes that are made public may enable others to predict the confidential information. The attribute inference problem is the possibility that data analyses could infer users' sensitive information $[19,14]$. Moreover, the malicious attacker has at its disposal all strategies that enable the compromise of users' privacy. However, for the work described here, we only consider a single data mining approach and we propose a privacy-preserving technique to provide privacy against such an attack. We first illustrate the privacy attack considered in this study.

\subsection{The Privacy Attack Model}

Consider an attacker $M$ who wants to infer the "Emotional Status" of an OSN user $u$ who regards this information as sensitive and whose OSN profile does 
not show it publicly. Here the user $u$ is considered as a target user. In order to launch the attack, $M$ can prepare a training data set $D_{t r}$ by analysing the OSN of a number $N$ of users, $A$ attributes and their friendship information. At first, $M$ may collect some information that $u$ discloses openly on their OSN profile (in this illustration we consider $u$ 's settings for the values of the "Hometown" and "Profession" a public view). $M$ then can select a set of users who disclose their "Emotional Status" information as well as the same information (i.e. "Hometown", "Profession") that user $u$ discloses on his/her profile. $M$ may store all this information in $D_{t r}$ and select "Emotional Status" as a class attribute and the rest of the attributes as non-class attributes.

Table 1. A hypothetical training data set.

\begin{tabular}{llll}
\hline User & Hometown & Profession & Class Attribute \\
\hline$a$ & Sydney & Entrepreneur & Lonely \\
\hline$b$ & Bathurst & Entrepreneur & Connected \\
\hline$c$ & Melbourne & Salesman & Connected \\
\hline$d$ & Sydney & Student & Lonely \\
\hline$e$ & Melbourne & Salesman & Connected \\
\hline
\end{tabular}

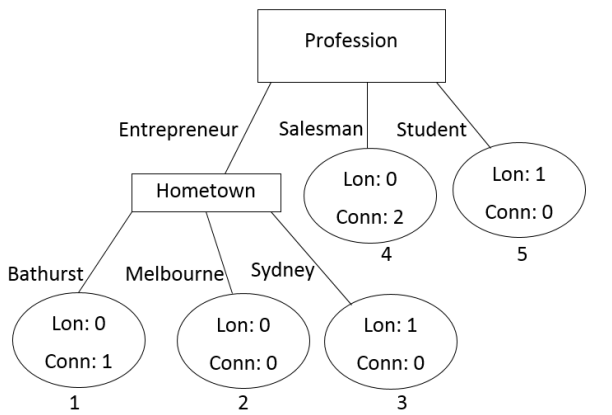

(a) Tree-1

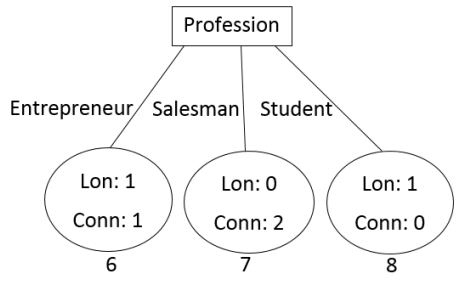

(b) Tree-2

Fig. 1. Decision trees build on the sample data set given in Table 1.

$M$ may include all users for building a training data set $D_{t r}$ that indicates clearly in their profile or recent posts that they are "Lonely" or "Connected". A sample of such training data set $D_{t r}$ is shown in Table 1 where rows are records and columns are attributes. This sample data set is an example of what could be prepared with any other set of attributes.

After preparing the training data set $D_{t r}, M$ may apply any data mining algorithms to obtain the rules (or patterns) from $D_{t r}$. Figure 1 presents a sample decision forest that can be generated from $D_{t r}$. The rectangular boxes in Figure 1 are called nodes and ovals are called leaves. The path between a root node (the node at the top) and a leaf presents a logic rule for the leaf. For example, the logic rule for Leaf 3 (see Figure 1(a)) states that if the attribute value of 
"Profession = Entrepreneur" and "Home town = Bathurst" then the class value is "Connected", and 1 record is in the leaf 1 having the class value "Connected". Here in this logic rule the condition Entrepreneur=Profession and "Bathurst= Home town" is called the antecedent of the logic rule and "Connected is called the consequent. By applying the rules derived from the data set containing the information about $u$, shown in Table 2, $M$ can predict the "Emotional Status" of $u$.

Table 2. A hypothetical test data set.

\begin{tabular}{llll}
\hline User & Hometown & Profession & Class Attribute \\
\hline$u$ & Bathurst & Student & $?$ \\
\hline
\end{tabular}

The existing privacy preserving technique [5] can preserve user $u$ 's privacy by suggesting $u$ to suppress some attribute values that appear most frequently in the logic rules. In this example, the attribute "Profession" appears in all the logic rules as shown in Figure 1. If the value of "Profession" is suppressed then all the logic rules (shown in Figure 1) will no longer be applicable to predict u's "Emotional Status". This technique appears to be effective to protect user's privacy however it does not consider the friendship information.

OSN is also known as social attribute network $(S A N)$. In an $S A N$ model, both users and their attribute-values are designed as vertices. Therefore, the attacker can take advantage of a metric function as shown in Equation 1 [1], to incorporate the friendship information into $D_{t r}$ in order to reveal the values of confidential attributes.

$$
m\left(u, A_{n}=v\right)=\sum_{t \in \Gamma_{s+}(u) \cap \Gamma_{s+}\left(A_{n}=v\right)} \frac{w(t)}{\log \left|\Gamma_{+}(t)\right|} .
$$

Here, $\Gamma_{s+}(u)$ is a set of OSN users connected to a user $u$ and $\Gamma_{s+}\left(A_{n}=v\right)$ is the set of users having the attribute-value $A_{n}=v$. Similarly, $\Gamma_{A_{n}+}(u)$ is the set of all attribute-value pairs linked to user $u$. Therefore, the neighbourhood of $u$ is represented as, $\Gamma_{+}(u)=\Gamma_{s+}(u) \cup \Gamma_{A_{n}+}(u)$. On the other hand, $t$ is the set of $u$ 's friends who have an attribute-value pair $A_{n}=v$ (i.e. $t \in \Gamma_{s+}(u) \cap \Gamma_{s+}\left(A_{n}=v\right)$ ) and $\Gamma_{+}(t)=\Gamma_{s+}(t) \cup \Gamma_{A_{n}+}(t)$. The $w(t)$ is the weight of each of them and its value is set to 1 in this study. The higher the value of $m\left(u, A_{n}=v\right)$ indicates the higher chance that $u$ has the value $v$ for attribute $A_{n}$. An interesting property of this metric is that, if friendship information is available, then $m\left(u, A_{n}=v\right)$ can be calculated for any attribute-value pair $A_{n}=v$ whether the user $u$ has that value or not.

To launch the attack using users' friendship information, $M$ can prepare a training data set $D_{t r}$ by storing $u$ 's available information (for example, Hometown and Profession) and friendship information calculated by using Equation 1 in it. We present a sample of such training data set in Table 3. The information directly related to OSN users (e.g. Hometown and Profession as shown in Table 3) 
Table 3. A hypothetical training data set with friendship information.

\begin{tabular}{lllllll}
\hline User Hometown & m_hometown & Profession & m_profession & m_emotional & Class Attribute \\
\hline$a$ & Sydney & 0 & Entrepreneur & 0 & 0.56 & Lonely \\
\hline$b$ & Bathurst & 0 & Entrepreneur & 0 & 0 & Connected \\
\hline$c$ & Melbourne & 0.56 & Salesman & 0 & 0 & Connected \\
\hline$d$ & Sydney & 0.51 & Student & 0.56 & 0.56 & Lonely \\
\hline$e$ & Melbourne & 0.51 & Salesman & 0 & 0 & Connected \\
\hline
\end{tabular}

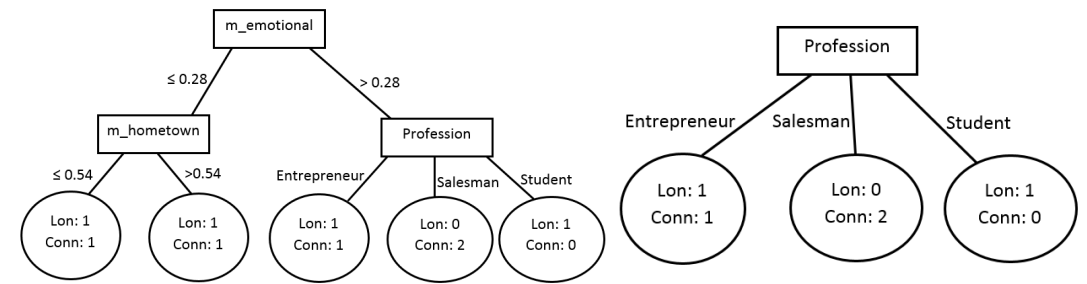

(a) Tree-1

(b) Tree-2

Fig. 2. Decision trees build on the sample data set given in Table 3.

are named regular attributes, whereas the information related to the friendship links (such as $\mathrm{m}_{-}$Hometown and $\mathrm{m} \_$Profession) are named link attributes.

$M$ can consider "Emotional Status" as the class attribute and then apply any machine-learning technique to obtain the patterns of the "Lonely" and "Connected" users from the data set. In Figure 2, we present a sample decision forest which can be built from $D_{t r}$ (as shown in Table 3). By using these rules, the attacker can predict the emotional status of a new user $u$ (shown in Table 4) even if the user does not disclose the information.

Table 4. A hypothetical test data set with friendship information.

\begin{tabular}{lllllll}
\hline User Hometown & m_hometown & Profession & m_profession & m_emotional & Class Attribute \\
\hline$u$ & Bathurst & 0.51 & Student & 0.51 & 0 & $?$ \\
\hline
\end{tabular}

We now argue that OSN users may have diverse preferences on what they consider sensitive. For example, one may consider their emotional status as sensitive only while others may consider their emotional status and/or political view as sensitive. Therefore, a privacy-preserving technique should be capable of protecting all the sensitive information considered by each user. At the same time, the techniques should be capable of providing privacy even if an adversary uses a different classifier (rather than the one used by the privacy preserving technique) to infer the class value of a target user.

Again, privacy is in a constant trade-off with personalization. The more a system (for example, a recommender system) knows about the user, the more it can tailor its services to the users. Therefore, In addition, while protecting users' privacy, a technique must consider maintaining the utility of a data set. A large number of attribute value suppression may provide better privacy, but 
at the same time, it also defeats the whole purpose of using social network sites for a user. The goal should be to provide privacy by suppressing the minimum number of attribute values.

\subsection{Related Work}

The existing privacy preserving techniques such as NOYB [8], TOTAL_COUNT [5], CUM_SENSITIVITY [5], and 3LP [16] can protect users' privacy against the attribute inference attack. The basic concept of these techniques are quite similar i.e. to select and suppress users' attribute values which are high predictors of a sensitive attribute $[2,3]$.

A commercially available privacy technique NOYB [8] follows a random process to suppress attribute values. Rather than using any classifier to extract the patterns from the training data set, $N O Y B$ takes a random approach to select and suppress the regular attribute values in the testing data set. In an extreme scenario, NOYB may obfuscate all the regular attribute values for the sake of providing privacy. The random obfuscation may remove a user's regular attribute value from public view in an OSN which has no bearing on the sensitive attribute. This unnecessary blocking of a user's profile is undesirable for users but less harmful that the fact that predictors of sensitive information are left for public use. Note that users generally want some information to be public, and hence the goal of a technique is to obfuscate the minimal number of attribute values to reduce the predictability of sensitive information.

TOTAL_COUNT and CUM_SENSITIVITY [5] on the other hand identify the influential regular attribute values to suppress which are predictors of a sensitive information. These two techniques can suggest the target user to suppress values one by one until the privacy of a sensitive attribute is protected. The functionality of TOTAL_COUNT and CUM_SENSITIVITY are almost the same except the attribute ranking procedure. Both techniques were shown to be useful and outperform NOYB. That is, in order to achieve a security threshold $S_{t}$, the required number of attribute value suppression in the case of $N O Y B$ [8] is much higher than TOTAL_COUNT and CUM_SENSITIVITY [5].

The data of an OSN can be represented as a social attribute network $(S A N)$ model which integrates both users' friendship network and attribute information [20]. However, TOTAL_COUNT and CUM_SENSITIVITY do not consider the friendship network which can be useful to infer users' sensitive attribute. Using the friendship network an adversary can infer a user's private information $[16]$.

In order to preserve a user's confidential information from being inferred by their friendship information, Estivill-Castro et al. proposed an approach $[6,7]$. which suggests the target users to un-friend or befriend other users randomly. The number of deletion or addition of friends can often be extensive and the target user may not like such kind of random connection/disconnection.

Heatherly et al. show a Naïve Bayes classifier based protection technique [11], let's call this technique PrivNB for short, that can provide privacy by suppressing attribute values and deleting friendship links. The effect of PrivNB 
on data utility of the protected data set was not analysed. Another technique namely $3 L P[16]$ (three layer of protection) based on decision forests algorithms can protect privacy even if an attacker utilises friendship information(along with the regular attributes) to invade privacy.

Similar to TOTAL_COUNT and CUM_SENSITIVITY, the algorithm 3LP takes a similar approach to identify the influential attributes in its first layer to suppress predictive attribute values from a victim's profile (Layer 1). However, $3 L P$ goes further to suggests the victim shall delete existing friends from the friend list (Layer 2) and add new friends (Layer 3). 3LP can protect privacy for a sensitive attribute (considered by its user) on a single run. However, the OSN users may consider multiple information as sensitive rather than a single information. Hence, $3 L P$ does not consider the protection policy for multiple attributes on its single run.

$3 L P$ assumes the existence of a single sensitive attribute such as the "Political View" while in reality a user is likely to have multiple sensitive attributes such as the "Political View" and "Religious View". To protect the privacy of multiple sensitive attributes $3 L P$ could be applied multiple times, but every run of $3 L P$ would be isolated/independent. As a result, they can be counterproductive in the sense that one run (say to protect the "Political View") might suggest hiding a friendship information with another user while a subsequent run (say to protect "Religious View") might suggest disclosing the same friendship information resulting in the loss of protection of "Political View".

\subsection{Our Contributions}

We propose $3 L P+$ in this study which is an extension of the existing $3 L P$ algorithm [16]. $3 L P+$ aims to provide privacy for multiple sensitive attributes

Table 5. The major contributions of this study.

\begin{tabular}{|c|c|c|}
\hline $\begin{array}{l}\text { Contribution } \\
\text { Number }\end{array}$ & Description of the contributions & Section \\
\hline 1 & $\begin{array}{l}\text { We propose a privacy preserving technique, } \\
\text { namely } 3 \mathrm{LP}+\text {, that provides privacy for multiple } \\
\text { sensitive attributes considered by a user. }\end{array}$ & 2 \\
\hline 2 & $\begin{array}{l}\text { We implement the } 3 L P+\text { on two OSN data sets } \\
\text { and the experimental results indicate the superiority } \\
\text { of } 3 L P+\text { over an existing technique, even if the attacker uses } \\
\text { a set of different classifiers to invade privacy. }\end{array}$ & 4.2 \\
\hline 3 & $\begin{array}{l}\text { We also evaluate the data utility of two OSN } \\
\text { data sets after applying } 3 L P+\text { and compare the } \\
\text { results with the previous privacy preserving techniques. }\end{array}$ & 4.3 \\
\hline
\end{tabular}

through a co-ordinated approach as opposed to the isolated approach. It uses 
a matrix to store the history of any friendship being hidden or new friendship being created during a run to avoid a conflicting suggestion in a subsequent run. For example, if the $t$-th run suggests hiding a friendship of the victim user with another user (and the victim user actions on the suggestion), then the matrix stores that information so that a subsequent run does not suggest the victim user creating the friendship with the same user.

We have three major contributions in this paper and we summarise each of them including their corresponding section number in Table 5. Out of these three contributions, we have previously published Contribution 1 in the International Conference on Information Systems Security and Privacy [18]. In that conference paper we implement the $3 L P+$ on a synthetically generated OSN data set only whereas in this paper we implement the $3 L P+$ on a Facebook real users' dataset along with the synthetic data set (see Section 3.1).

The rest of this paper is organized as follows. Section 2 presents our proposed technique $3 L P+$. We describe the experimental set up in Section 3 and the experimental results in Section 4. Section 5 gives a concluding remark.

\section{The Proposed Technique}

The basic idea of $3 L P+$ is to protect the privacy of all information that a user considers to be sensitive. Users can give the list of attributes they considers sensitive and then, $3 L P+$ provides three steps (or layers) of recommendations:

Step 1: Compute the sensitivity of each attribute for each user and suggest to the user which attribute values the user needs to suppress. The pseudo-code for Step 1 is reproduced here within Algorithm 1 from [18], $3 L P+$ selects a class attribute (from the list of sensitive attributes considered by the $3 L P+$ User $u$ ) randomly, prepares a training data set $D$, and then applies SysFor [12] on $D$ to get a set of logic rules. $3 L P+$ then uses the support and confidence of each rule to compute its sensitivity (or Rule Sensitivity) value in breaching the privacy of a sensitive attribute. We set this threshold value to 1.006 in order to keep consistence with the previous studies [5, 16]. Similar to previous studies [16,17], the rules having Rule Sensitivity value 1.006 or above are considered Sensitive Rules in this study. We utilize the function GetSensitiveRules() in Step 2 of Algorithm 1 to represent the processes of generating the sensitive rule set $R^{u}$ for $u$.

After preparing the sensitive rule set $R^{u}, 3 L P+$ counts the number of appearance of each regular attribute $A_{n}$ in $R^{u}$ and store $A_{n}$ in $A^{s}$. Here, $A^{s}$ stores all the regular attributes and the number of their appearances in $R^{u}$. One attribute can appear only once in a sensitive rule $R_{j}^{u}$ but more than once in $R^{u}$. The regular attribute $A_{n}$ with the highest number of appearances in $R^{u}$ is suggested to $u$ for suppression. The decision is then up to $u$ whether to suppress its value or not. If $u$ suppresses the value of $A_{n}$, then $A_{n}$ is no longer available in $A^{s}$ and all sensitive rules in $R^{u}$ that have $A_{n}$ in their antecedent are no longer applicable for $u$. Regardless whether $u$ suppresses the attribute or not, $3 L P+$ then identifies $A_{n}$ with the next highest appearances and suggests $u$ to suppress that. The process continues until $R^{u}$ or $A^{s}$ becomes empty. 
Step 2:Hide friendship links as necessary if they are not fabricated previously.

After Layer 1, if any sensitive rule remains in $R^{u}$ such a rule only uses link attributes (i.e. the attribute values can only be altered by using Eq. 1). Therefore, if a link attribute appears as an antecedent of a sensitive rule $R_{j}^{u}$ (i.e. $R_{j}^{u} \in R^{u}$ ), where the value of the link attribute needs to be greater than a constant SplitPoint (as mentioned in $R_{j}^{u}$ ), the $3 L P+$ explores to reduce its value $<$ SplitPoint by hiding some of $u$ 's friendship links. By doing this $3 L P+$ makes the rule unusable to predict the class value of $u$ with certainty.

In Step 2, 3LP+ first identifies the link attribute, $A_{n}$, that appears most in the sensitive rule set $R^{u}$ and compute $A_{n}$ 's value, denoted as $V$, using Eq. 1 . If $V$ is higher than the split point mentioned in $R_{j}^{u}$, then $3 L P+$ suggests $u$ to hide a friendship link. While choosing a friendship link, $3 L P+$ selects a friend, $t_{i}$, of $u$ who has the smallest degree and has not previously appeared in the friendship matrix $F$ (here $F$ is an $1 \times N$ matrix which stores the Flag information for $u$ ). The $3 L P+$ recommends $u$ to hide $t_{i}$ so that it can reduce $V$ 's value the most by hiding a minimum number of friends. If $u$ follows the recommendation, $3 L P+$ puts a Flag up in the $i^{t h}$ column of the friendship matrix $F$ and this ex-friend will not be recommended for further hiding or adding. $3 L P+$ then updates $G^{\prime}, F^{\prime}$, and recomputes $V$ 's value.

This process continues until the value $V$ is lower than the SplitPoint mentioned in $R_{j}^{u}$. Once the $V$ is lower than the split point, then the process of hiding friends stops and $3 L P+$ removes $R_{j}^{u}$ and other rules (which have an antecedent with the value $V$ ) from $R^{u}$ as they are no longer be applicable to determine $u$ 's class value. At the end of Step 2 , if $R^{u}$ is not empty then only $3 L P+$ moves to Step 2 i.e. Layer 3.

Step 3: Add friendship links as necessary if they are not fabricated previously.

After Step 1 and Step 2, any sensitive rule remains in $R^{u}$, that contains link attribute only and tests for a value $V \leq$ some SplitPoint in its antecedent. In this case, $3 L P+$ suggests $u$ to add new friends so that the $V$ becomes greater than the split point in $R_{j}^{u}$ and thus $R_{j}^{u}$ is no longer applicable to $u$. While adding any friend on $u$ 's friend list, a user $t_{i}$ is selected in such a way that a Flag has not been up previously in the $i^{\text {th }}$ column of $F^{\prime}$ matrix and having the smallest $\Gamma_{+}(t)$ value. If $u$ accepts the recommendation, $3 L P+$ then updates the matrix $F^{\prime}$, friendship graph $G^{\prime}$, and $V$ increases.

This adding process continues until the value $V$ exceeds the split point value. It is noted that, adding a new friend on a profile is complicated and the fact that two users share the friendship that is not the ownership of either alone [9]. depends on the other users to confirm the friendship on OSN. Hence, $3 L P+$ keeps these recommendations as a last resort. Our experimental results also indicate this step is seldom required. 


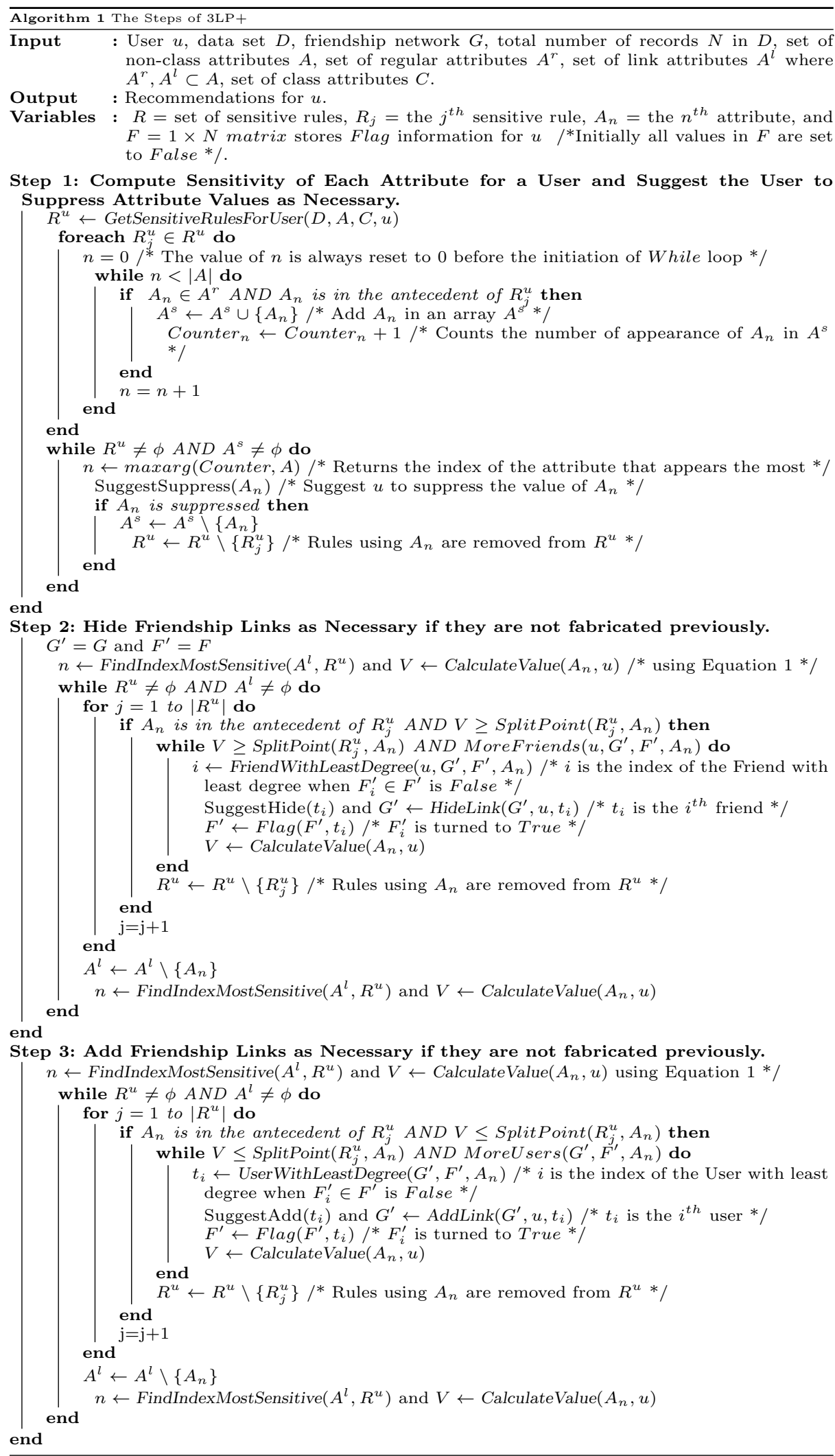




\section{Experiments}

\subsection{Data Set}

We implement the privacy preserving techniques on two OSN data sets. The first data set [15], denoted as $D_{1}$, contains 1000 records, 11 regular attributes, and 50,397 friendship links among the users. The second data set, denoted as $D_{2}$, is prepared by gathering the users' information from Facebook. $D_{2}$ contains 616 records, 24 regular attributes, and 1280 friendship links among the users. In order to insert the users' link attribute values into the data set, we calculate metric values for each regular attribute (using Equation (1)) and therefore, the total number of attributes becomes 22 (i.e. 11 regular attributes and 11 link attributes) for $D_{1}$ and 48 attributes for $D_{2}$.

\begin{tabular}{|c|c|c|c|}
\hline Test data set & $D_{t s, X}$ & $D_{t s, Y}$ & $D_{t s, Z}$ \\
\hline $\begin{array}{c}\text { Group } 1 \\
\text { (6\% records) }\end{array}$ & $\mathrm{X}: 2 \%$ records & $\mathrm{Y:}: 2 \%$ records & Z: $2 \%$ records \\
\hline \multirow{2}{*}{$\begin{array}{c}\text { Group } 2 \\
\text { (3\% records) }\end{array}$} & $\mathrm{X}, \mathrm{Y}: 1 \%$ records & $\mathrm{X}, \mathrm{Y}: 1 \%$ records & $\mathrm{Y}, \mathrm{Z}: 1 \%$ records \\
\hline & $X, Z: 1 \%$ records & $\mathrm{Y}, \mathrm{Z}: 1 \%$ records & $X, Z: 1 \%$ records \\
\hline $\begin{array}{c}\text { Group } 3 \\
\text { (1\% records) }\end{array}$ & \multicolumn{3}{|c|}{$X, Y, Z: 1 \%$ records } \\
\hline
\end{tabular}

Fig. 3. Distribution of $10 \%$ test data set records in a fold. Here the different colours indicate the different records and same colour indicates the same records.

We assume the users in the two data sets consider three attributes as sensitive. For the simplicity let's denote them as $X, Y$, and $Z$. In the $D_{1}$ data set, the sensitive attributes are: $X=$ "Political view", $Y=$ "Religious view", and $Z=$ "Sexual orientation". In the $D_{2}$ data set, the sensitive attributes are: $X=$ "Emotional status", $Y=$ "Religious view", and $Z=$ "Political view".

We therefore prepare three versions of each data set for each of the sensitive attribute and in each version we consider a sensitive attribute as a class attribute. We follow 10-fold cross validation method through out our experiments. Therefore, in each fold, a training data set, denoted as $D_{t r}$, contains $90 \%$ of the total records and a testing data set, denoted as $D_{t s}$, contains $10 \%$ of the total records.

For the experimental purpose, we split the $10 \%$ test data records into three groups: Group 1 ( $6 \%$ records), Group 2 (3\% records), and Group 3 ( $1 \%$ records). We assume the Group 1 users consider any one attribute (i.e. either " $X$ " or " $Y$ " 
or " $Z$ ") as sensitive. On the other hand, we assume Group 2 users consider any two attributes (out of the three attributes) as sensitive. Finally, Group 3 consists of $1 \%$ users who consider all the three attributes as sensitive. We present the three groups and their records distribution in Figure 3. Here the different cell colours indicate different records and the same colour represents the same records.

While preparing a test data set e.g. $D_{t s, X}$ we select the records who consider $X$ as a sensitive information and return all other records in the training data set $D_{t r, X}$. For example, in case of the first data set $D_{1}$, there are 100 records (out of 1000 total records) in a test data set (after applying 10-fold cross validation). We only keep 50 records in $D_{t s, X}$ who consider $X$ as sensitive and return rest of the 950 records in the training data set $D_{t r, X}$. Similarly, for the second data set $D_{2}$, we keep 31 records in $D_{t s, X}$ and 585 records in $D_{t r, X}$. On the other hand, when we consider a particular attribute as a class attribute then rest of the attributes are considered to be non-class attributes. For example, in $D_{t r, X}$ and $D_{t s, X}$ data sets, both $Y$ and $Z$ are selected as non-class attributes.

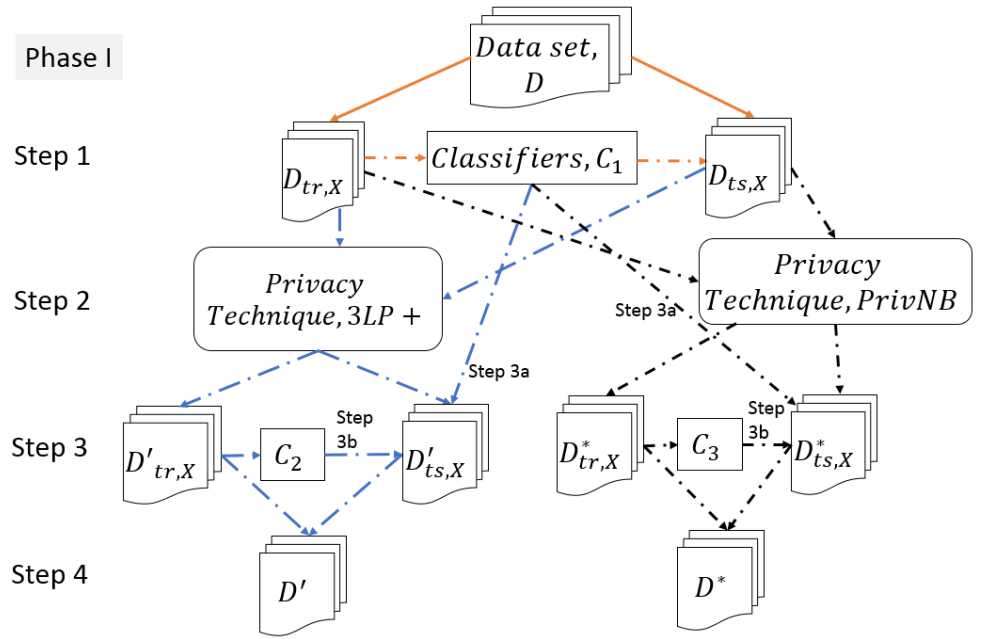

Fig. 4. Phase I of the experiments.

\subsection{Experimental Set-up}

We present the entire experimental set-up in three phases for three different sensitive attributes. In Phase I, we first protect the privacy for $X$, then for $Y$ in Phase II, and finally, for $Z$ in Phase III. We argue that the $3 L P+$ can protect privacy of all the sensitive information (which are selected by its users) regardless to the sequence of selection as a class attribute. Therefore, we also conduct experiments in an opposite sequence order but for simplicity we only describe the experimental set-up here for first sequence order. 

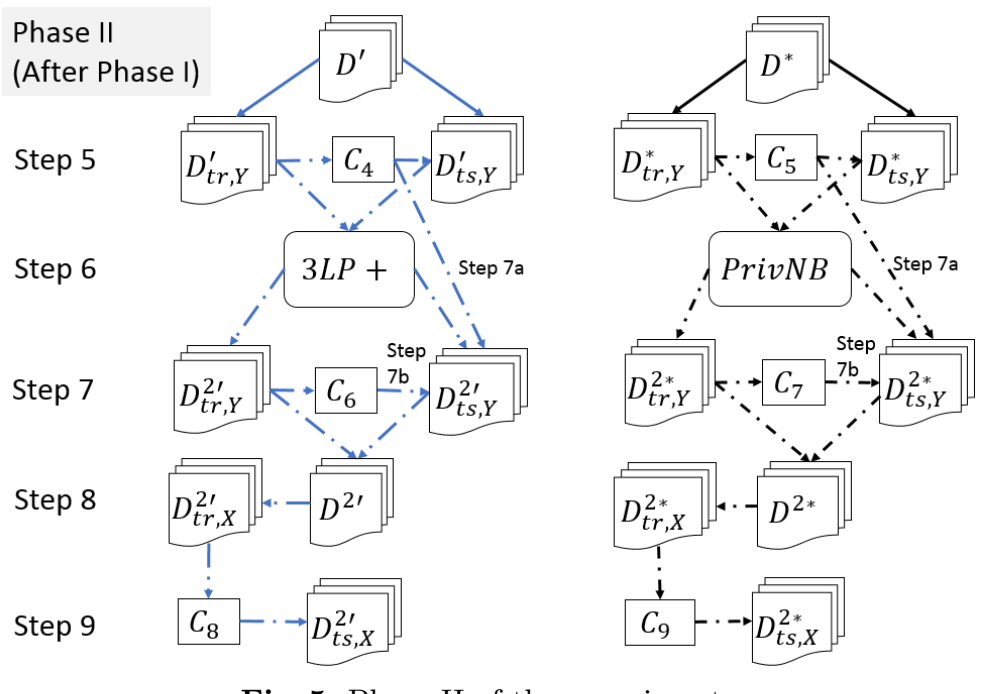

Fig. 5. Phase II of the experiments.

Phase I During the first step, illustrated in Figure 4 taken from [18], we prepare a training data set $D_{t r, X}$, and a testing data set $D_{t s, X}$ from the main data set $D$ by considering users' $X$ as a class attribute. At Step 2, we apply the two privacy preserving techniques, i.e. $3 L P+$ and PrivNB, on the insecure test data sets. Here the term 'insecure' means that the $3 L P+$ or PrivNB have not been applied previously on the test data sets and hence the users' class value can be determined by an attacker easily. The test data sets are then secured by the techniques, as shown in Step 3, denoted as $D_{t s, X}^{\prime}$ and $D_{t s, X}^{*}$ respectively. We calculate and compare the number of insecure users exists in the insecure and secure data sets. In order to provide privacy $3 L P+$ and PrivNB modifies the data sets by hiding information/friends or adding friends. Therefore, we use two different symbols ' and $*$ throughout the experimental set-up to denote the modified data sets by $3 L P+$ and PrivNB techniques respectively.

A privacy provider may not determine the classifier which is going to be used by an attacker and therefore, the privacy protecting technique should be able to protect privacy against any machine learning algorithms. In our experiments we explore and compare the performance of $3 L P+$ and PrivNB for different classifiers such as Naïve Bayes classifier $(N B)$, Support Vector Machine $(S V M)$, and Random Forest $(R F)$ algorithm. In order to do that we first apply these machine learning algorithms on insecure data set $D_{t s, X}$ in Step 1 and find the number of number of insecure users in the test data set. We name it as classifiers' accuracy which refers to the number of users whose class value is identified by the classifiers. The larger the accuracy value indicates lower the privacy.

We then apply the different classifiers on secure test data sets $D_{t s, X}^{\prime}$ and $D_{t s, X}^{*}$ in Step 3. By comparing the classifiers' accuracy results, in Step 1 and Step 3, we then determine which technique provides better privacy on the test data sets. The results are presented in terms of number of insecure users, denoted as $t_{0}^{s}$ and 


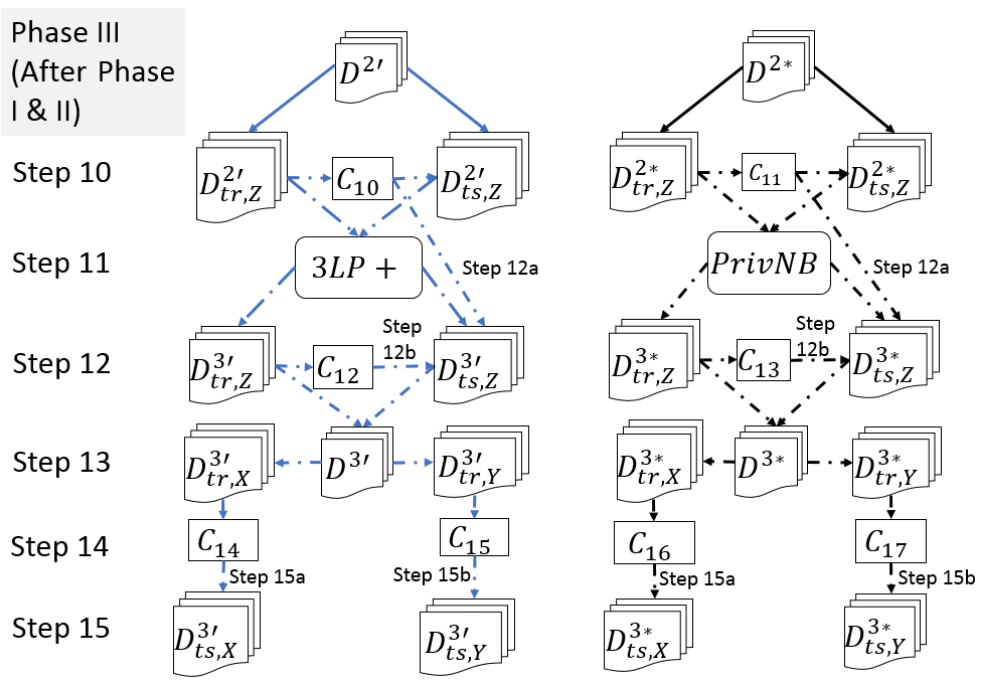

Fig. 6. Phase III of the experiments.

classifiers' accuracy, denoted as $t_{0}^{c_{1}}$. In Step 3, we also calculate data utility (in terms of number of suppressed attribute values) in $D_{t s, X}^{\prime}$ and $D_{t s, X}^{*}$ after applying the two privacy preserving techniques and denoted as $t_{3}^{u}$. We have taken Figs. 4- 6 from [18]; where different colours of arrows indicate the procedure of two different privacy preserving techniques.

Phase II After Phase I, we select $Y$ as a class attribute (refer to Figure 5 taken from [18]). We first prepare training and testing data sets and denote them as $D_{t r, Y}^{\prime}$ and $D_{t s, Y}^{\prime}$ which are prepared from $D^{\prime}$. Similarly, $D_{t r, Y}^{*}$ and $D_{t s, Y}^{*}$ are prepared from $D^{*}$. In Step 5, different classifiers are applied on $D_{t s, Y}^{\prime}$ and $D_{t s, Y}^{*}$, denoted by $C_{4}$ and $C_{5}$ respectively, to measure the classifiers' accuracy. Then we apply $3 \mathrm{LP}+$ and PrivNB on test data sets in Step 7 (in order to secure the users' privacy). Similar to Phase I, the experimental results are analysed and compared in terms of number of insecure users, data utility, and prediction accuracy by different classifiers in Step 7.

After Step 7 we again return all the records to original data set and thus it is modified to $D^{2^{\prime}}$ and $D^{2 *}$ for $3 L P+$ and PrivNB respectively. In Step 9, we again investigate the safety of users (who considered $X$ as sensitive) in $D_{t s, X}^{\prime}$ due to providing the privacy to users in $D_{t s, Y}^{*}$.

Phase III We select $Z$ as a class attribute in this phase and similar to previous two phases, we first prepare training and testing data sets i.e. $D 2^{\prime}{ }_{t r, Z}$ and $D 2^{\prime}{ }_{t s, Z}$ (refer to Figure 6 taken from [18]) from $D^{2^{\prime}}$. We also prepared $D_{t r, Z}^{2 *}$ and $D_{t s, Z}^{2 *}$ are prepared from $D^{2 *}$. In Step 10, we apply different classifiers, denoted by $C_{10}$ and $C_{11}$, on the two test data sets $D_{t s, R}^{2^{\prime}}$ and $D_{t s, R}^{2 *}$ to find the classification accuracy before and applying any privacy techniques. We then apply $3 L P+$ on $D_{t s, R}^{2^{\prime}}$ and PrivNB on $D_{t s, R}^{2 *}$ in Step 11. We denote $D_{t s, R}^{3^{\prime}}$ and $D_{t s, R}^{3 *}$ to represent the secure test data sets and apply different classifiers, 
denoted by $C_{10}$ and $C_{11}$, again on them in Step 12. After securing the test data sets, similar to Phase I and Phase II, we again analyse and compare the number of insecure users (in $D_{t s, X}^{3^{\prime}}, D_{t s, X}^{3 *}, D_{t s, Y}^{3 *}, D_{t s, Y}^{3 *}$ ) in Step 15a and Step 15b as shown in Figure 6. The secured test data sets are analysed and compared in terms of number of insecure users and data utility for both privacy preserving techniques.

\section{Results and Discussion}

We present the experimental results of our proposed technique $3 L P+$ in this section. The results are averaged before we present here and then compare with an existing technique PrivNB [11]. The results are shown using bar graphs where $x$-axis represents the step numbers mentioned in Section 3.2 and $y$-axis represents the Attack Success Rate percentage. Here the term Attack Success Rate indicates the number of users whose class value is correctly predicted by the classifier. Higher Attack Success Rate percentage indicates the greater chance for the intruder to infer the class value of a user and vice versa.

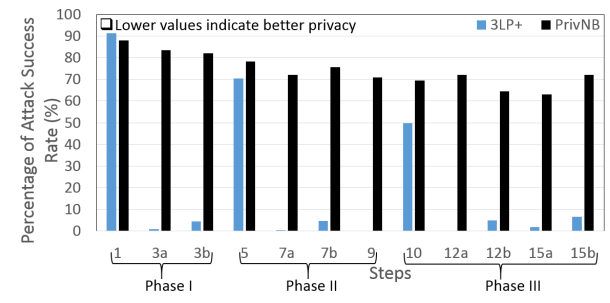

(a) Dataset $1, D_{1}$

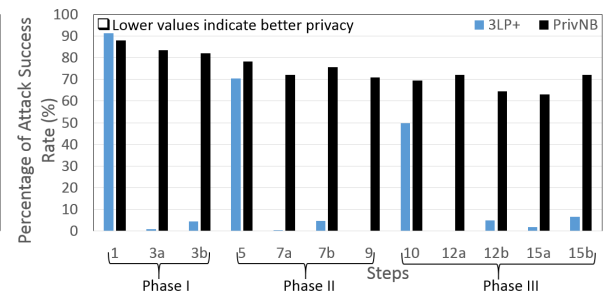

(b) Dataset 2, $D_{2}$

Fig. 7. Prediction of class value accuracy of two privacy preserving techniques.

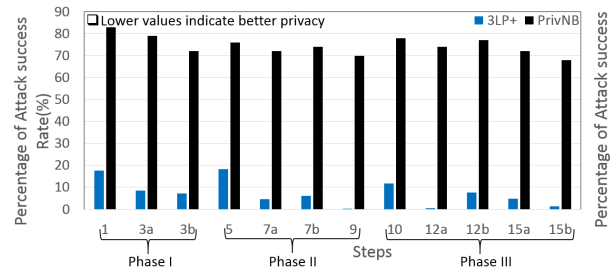

(a) Dataset $1, D_{1}$

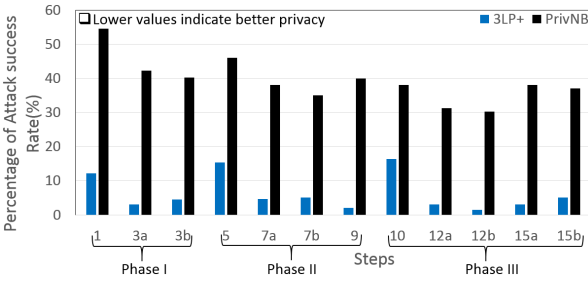

(b) Dataset 2, $D_{2}$

Fig. 8. Prediction of users' class value(correctly) using the same classifier used by the privacy preserving techniques.

\subsection{Protection against the Same Classifier}

In Figure 7 we present the number of insecure users whose class value can still be inferred by applying the same classifier used by the privacy protection technique. 
As mentioned earlier, 3LP+ uses Sysfor [12] decision forests and PrivNB uses Naïve Bayes classifier to extract the patterns from the data set. We first provide privacy by the two privacy techniques separately as described in the Section 3.2. Here $y$-axis represents prediction probability to classify a record (regardless to correctly or incorrectly classified) by an intruder after the protection techniques are applied. We observe that the probability percentage of records is much higher for PrivNB compared to $3 L P+$ except at Step 1 . This is because the privacy preserving techniques are yet to implement at Step 1 as shown in Figure 4. On the other hand, in Figure 8(a), the percentage of correctly classified records by PrivNB is approximately $70 \%$ more than the $3 L P+$. We observe a similar results in Figure 8(b) for the second data set $D_{2}$. We also explore the performance of the two privacy protection techniques against different classifiers (not used by the privacy protection technique) and the results are shown in next section.

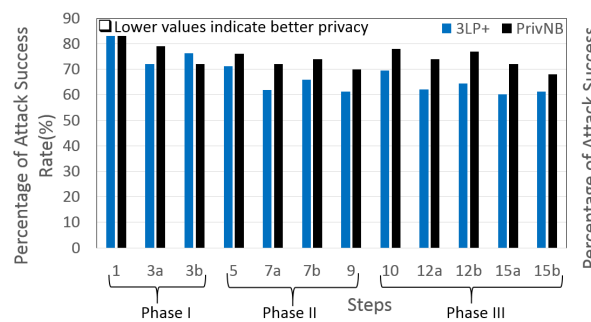

(a) Dataset $1, D_{1}$

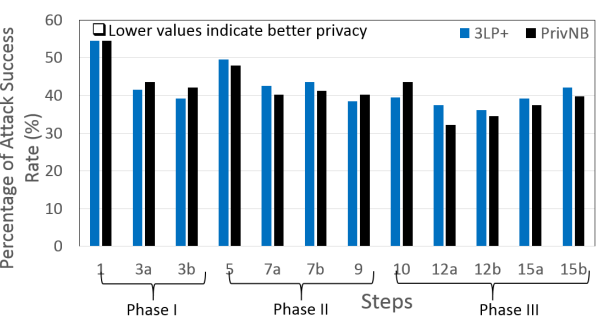

(b) Dataset 2, $D_{2}$

Fig. 9. Performance of Naïve Bayes in order to breach users' privacy in the test data sets.

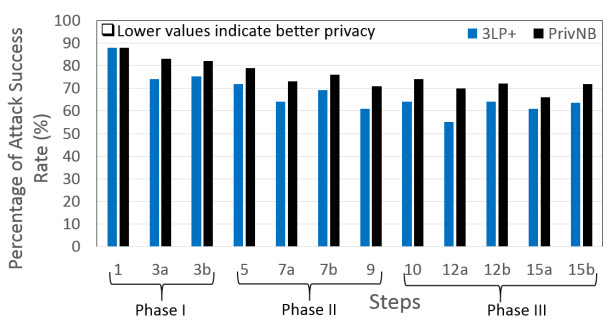

(a) Dataset $1, D_{1}$

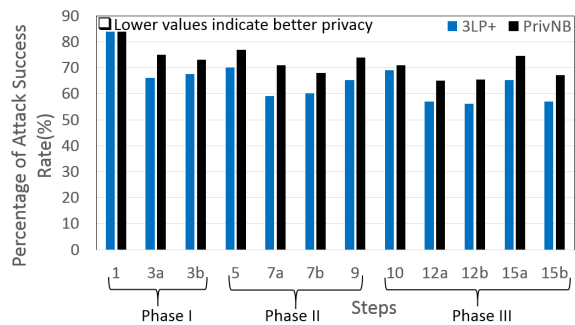

(b) Dataset 2, $D_{2}$

Fig. 10. Performance of Support Vector Machine in order to breach users' privacy in the test data sets.

\subsection{Protection against Different Classifiers}

In order to explore the performance of the two privacy protection techniques, we utilise three conventional classifiers that the attacker may use to invade privacy 
and they are: Naïve Bayes (denoted as NB), Support Vector Machine (denoted as $S V M$ ), and Random Forest [4] (denoted as RF). We use WEKA [10] to implement the classifiers in our experiments. In Figure 9 we present the results of $3 L P+$ and PrivNB if the attacker uses $N B$ classifier to infer the sensitive attributes. We observe in Figure 9(a) (in $D_{1}$ data set) that the provided privacy by $3 L P+$ is better than PrivNB as the classification accuracy drops on average 10 percent compared to Step 1 to Step 3. However, this accuracy drops is less than 10 percent in case of PrivNB. The similar trend is observed throughout the experimental steps except Step 1. This is because at step 1 the privacy technique was yet to apply on the test data set. Therefore, classification accuracy is similar for $3 L P+$ and PrivNB at this step. For rest of the experimental steps, $3 L P+$ outperforms PrivNB.

In $D_{2}$ data set, shown in Figure 9(b), PrivNB performs a bit better than $3 L P+$. Unlike $3 L P+$, PrivNB uses Naïve Bayes classifier to extract the pattern from the training data set. Therefore it gets an advantage to suppress the highly predictor attribute values as Naïve Bayes consider to correctly predict the class value of a record.

We present the performance of the privacy preserving techniques against $S V M$ and $R F$ in Figure 10 and Figure 11 respectively. Our proposed technique clearly outperforms the existing PrivNB technique in both data sets and we can see a Attack Success Rate drop for both SVM and RF classifiers.

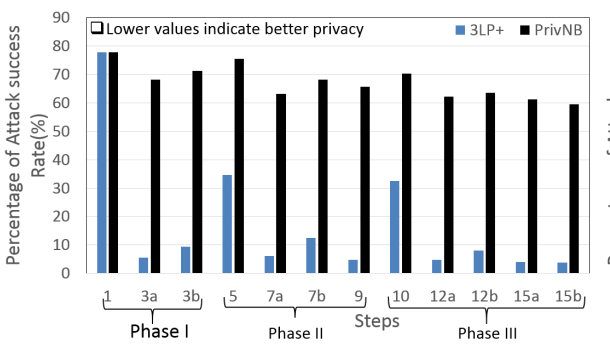

(a) Dataset $1, D_{1}$

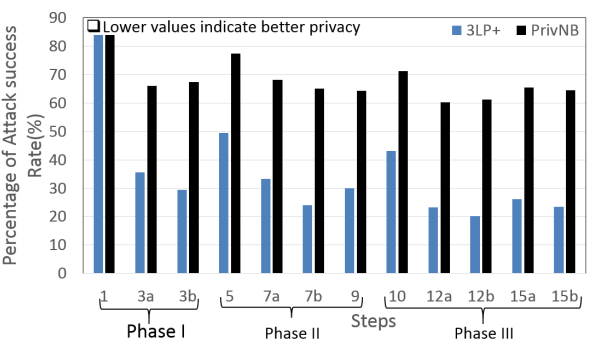

(b) Dataset 2, $D_{2}$

Fig. 11. Performance of Random Forest Algorithm in order to breach users' privacy in the test data sets.

We observe the prediction accuracy of the classifiers are decreasing as the steps are progressing. The reason is as the number of suppressed attribute values are increasing (to secure from sensitive rules) as the steps are progressing. It is important to note that the classifiers do not consider a missing value in a test data set While predicting a class value and thus reduce the probability for correct classification. We mentioned earlier that the link attribute values can only be modified. Thus it increases the possibility to classify a record correctly by both $S V M$, and $R F$. However, the $3 L P+$ still performs better in all steps compared to PrivNB. It is also noted that we do not consider the attribute Gender 


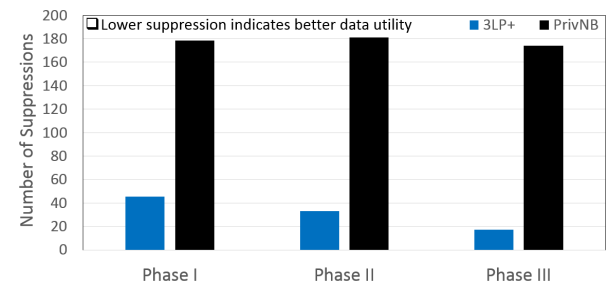

(a) Dataset $1, D_{1}$

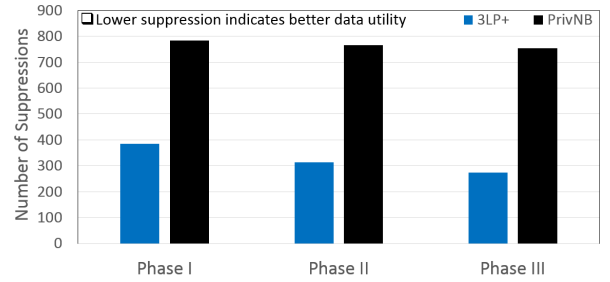

(b) Dataset 2, $D_{2}$

Fig. 12. Comparison of attribute value suppression.

for suppression in any of the test data sets as We believe Gender can be inferred from the user's name or photo.

\subsection{Data Utility}

We measure the data utility of the test data sets in terms of number of suppressed attribute values. The number of suppressions in test data sets after applying two different privacy preserving techniques are then compared with each other. In Figure 12 we present and compare the results for Step 3, Step 7, and Step 12 only as the privacy techniques are applied in these steps.

In first data set $D_{1}$, each test data set contains maximum 500 regular attribute values (i.e. 50 records $\times 10$ regular attribute values $=500$ ) before applying any privacy privacy preserving techniques. However, some of the records may consider more than one sensitive attribute (as shown in Figure 3) so, the number of attribute values are varied in different test data sets. As an example, if there are 100 records in a test data set $D_{t s, X}$, then 20 users consider a single attribute (i.e. $X$ ) as sensitive, 20 more users consider any 2 attributes as sensitive, and 10 other users consider all the 3 attributes as sensitive. Therefore, the maximum available number of attribute values in $D_{t s, X}$ is $(20 * 10+20 * 9+10 * 8=460)$. However, this number is not same for rest of the two test data sets i.e. $D_{t s, Y}$, and $D_{t s, Z}$. Because after applying $3 L P+$ or PrivNB on $D_{t s, X}$, the available number of attribute values will be different on $D_{t s, Y}$, and $D_{t s, Z}$, but it must not exceed the 460 .

Similar to $D_{1}$ data set, each test data set in $D_{2}$, contains maximum 1457 regular attribute values (i.e. 31 records $\times 47$ regular attribute values $=1457$ ) before applying any privacy privacy preserving techniques. However, this number is not the same for rest of the test data sets. In Figure 12(a) we observe the number of suppressed attribute values by the PrivNB technique is almost three times higher than $3 L P+$. We see a similar pattern in Figure 12(b) where the number of suppressions for PrivNB is much higher than $3 \mathrm{LP}+$.

\section{Conclusion}

We propose $3 L P+$ in this study to provide users' privacy on social media. Previous privacy preserving techniques can protect users' single sensitive attribute (from being inferred) whereas $3 L P+$ takes a coordinated approach to 
protect users' multiple sensitive attributes in one run. Our experimental results indicate that $3 L P+$ can provide better privacy compared to an existing privacy preserving technique by suppressing less number of attribute values compared to an existing technique. Our experimental results also show that $3 L P+$ can maintain the high utility of the data set by suppressing less number of attribute values compared to an existing technique.

In this paper, we have considered that an attacker uses Naïve Bayes, Support Vector Machine, Random Forest classifiers to invade privacy. If the attacker applies a different set of decision forest algorithms or classifiers to learn the patterns of the data set, then the calculation will be different and it is kept as a direction for further investigation.

\section{References}

1. Adamic, L.A., Adar, E.: Friends and neighbors on the web. Social networks 25(3), 211-230 (2003)

2. Al-Saggaf, Y., Islam, M.Z.: Privacy in social network sites (sns): The threats from data mining. Ethical Space: Int. J. Communication 9(4), 32-40 (2012)

3. Al-Saggaf, Y., Islam, M.Z.: Data mining and privacy of social network sites users: implications of the data mining problem. Science and engineering ethics 21(4), 941-966 (2015)

4. Breiman, L.: Random forests. Machine learning 45(1), 5-32 (2001)

5. Estivill-Castro, V., Hough, P., Islam, M.Z.: Empowering users of social networks to assess their privacy risks. In: Big Data (Big Data), 2014 IEEE International Conference on. pp. 644-649. IEEE (2014)

6. Estivill-Castro, V., Nettleton, D.F.: Can on-line social network users trust that what they designated as confidential data remains so? In: Trustcom/BigDataSE/ISPA, 2015 IEEE. vol. 1, pp. 966-973. IEEE (2015)

7. Estivill-Castro, V., Nettleton, D.F.: Privacy tips: Would it be ever possible to empower online social-network users to control the confidentiality of their data? In: Proceedings of the 2015 IEEE/ACM International Conference on Advances in Social Networks Analysis and Mining 2015. pp. 1449-1456. ACM (2015)

8. Guha, S., Tang, K., Francis, P.: Noyb: Privacy in online social networks. In: Proceedings of the first workshop on Online social networks. pp. 49-54. ACM (2008)

9. Gürses, G., Berendt, B.: The social web and privacy: Practices, reciprocity and conflict detection in social networks. In: Privacy-Aware Knowledge Discovery, Novel Applications and New Techniques. pp. 395-429. CRC Press (2010)

10. Hall, M., Frank, E., Holmes, G., Pfahringer, B., Reutemann, P., Witten, I.H.: The weka data mining software: an update. SIGKDD Explorations 11(1), 10-18 (2009)

11. Heatherly, R., Kantarcioglu, M., Thuraisingham, B.: Preventing private information inference attacks on social networks. IEEE Transactions on Knowledge and Data Engineering 25(8), 1849-1862 (2013)

12. Islam, Z., Giggins, H.: Knowledge discovery through sysfor: a systematically developed forest of multiple decision trees. In: Proceedings of the Ninth Australasian Data Mining Conference-Volume 121. pp. 195-204. Australian Computer Society, Inc. (2011)

13. Kosinski, M., Stillwell, D., Graepel, T.: Private traits and attributes are predictable from digital records of human behavior. Proceedings of the National Academy of Sciences 110(15), 5802-5805 (2013). https://doi.org/10.1073/pnas.1218772110 
14. Mislove, A., Viswanath, B., Gummadi, K.P., Druschel, P.: You are who you know: inferring user profiles in online social networks. In: Proceedings of the third ACM international conference on Web search and data mining. pp. 251-260. ACM (2010)

15. Nettleton, D.F.: Generating synthetic online social network graph data and topologies. In: 3rd Workshop on Graph-based Technologies and Applications (Graph-TA), UPC, Barcelona, Spain (2015)

16. Reza, K.J., Islam, M.Z., Estivill-Castro, V.: 3lp: Three layers of protection for individual privacy in facebook. In: IFIP International Conference on ICT Systems Security and Privacy Protection. pp. 108-123. Springer (2017)

17. Reza, K.J., Islam, M.Z., Estivill-Castro, V.: Social media users' privacy against malicious data miners. In: Intelligent Systems and Knowledge Engineering (ISKE), 2017 12th International Conference on. pp. 1-8. IEEE (2017)

18. Reza, K.J., Islam, M.Z., Estivill-Castro, V.: Privacy preservation of social network users against attribute inference attacks via malicious data mining. In: Proceedings of the 5th International Conference on Information Systems Security and Privacy - Volume 1: ICISSP. pp. 412-420. INSTICC, SciTePress (2019). https://doi.org/10.5220/0007390404120420

19. Ryu, E., Rong, Y., Li, J., Machanavajjhala, A.: Curso: protect yourself from curse of attribute inference: a social network privacy-analyzer. In: Proceedings of the ACM SIGMOD Workshop on Databases and Social Networks. pp. 13-18. ACM (2013)

20. Yin, Z., Gupta, M., Weninger, T., Han, J.: Linkrec: a unified framework for link recommendation with user attributes and graph structure. In: Proceedings of the 19th international conference on World wide web. pp. 1211-1212. ACM (2010) 